\title{
Realization of a room-temperature/self-powered humidity sensor, based on $\mathrm{ZnO}$ nanosheets
}

\author{
E. Modaresinezhad and S. Darbari ${ }^{1}$ \\ ECE Department, Tarbiat Modares University, Tehran, Iran
}

\begin{abstract}
In this paper, we report the first use of $\mathrm{ZnO}$ nanosheet-based direct-current nanogenerators (NG), as a room-temperature self-powered humidity sensor. It has been shown that the networked $\mathrm{ZnO}$ nanosheets are structurally stable under external mechanical pressures; hence these 2D nanostructures can be proposed as an emerging/attractive candidate in the field of mechanical energy harvesting. Also, benefiting from the high surface adsorption activity and large surface-to-volume ratio of $\mathrm{ZnO}$ nanosheets, we propose the fabricated NG as a promising self-powered gas sensor. Our study demonstrates short circuit and open circuit sensitivities of about $43.4 \%$ and $-2.96 \%$, because of humidity exposure. It is notable that the achieved output short circuit sensitivity is more than 10 times higher than the maximum sensitivity of a recently reported self-powered humidity sensor, based on $\mathrm{ZnO}$ nanorods. High sensitivity and stability, as well as direct-current output current are attractive properties of the realized humidity sensor, which can be proposed as a promising selfpowered sensor.
\end{abstract}

\section{Keywords—ZnO nanosheets;Nanogenerator;gas sensor;Self-powered sensor}

\section{INTRODUCTION}

It is well established that metal-oxide nanostructures have high surface to volume ratio, stability and surface adsorption activity, which entitles them as attractive candidates for gas sensors [1,2]. Among metal-oxides, $\mathrm{ZnO}$ nanostructures benefit from coupled semiconducting/piezoelectric properties which can be applied to realize a new generation of self-powered gas sensors. Since 2006, ZnO nanowires (NW) have been introduced as active components of piezoelectric nanogenerators (NGs), which convert mechanical energy to electricity [3-11]. Piezoelectric NGs can potentially convert different mechanical sources, including: (i) mechanical-movement energy, such as body or muscle movement [12-17]; (ii) vibration energy, from acoustic or ultrasonic waves [3-6]; (iii) and hydraulic energy, such as the flow of body fluids or blood, or dynamic fluids in nature [18]. As the most ideal

\footnotetext{
${ }^{1}$ Corresponding author, Tel.: +982182883905; fax:+982182884325.
}

E-mail address: s.darbari@modares.ac.ir (S. Darbari). 
application of NGs, it is desired that piezoelectric NGs will be applicable to power implantable devices such as pacemaker [19], remote patient monitoring or environmental monitoring. In this line of research, "self-powered devices" were firstly proposed by Xu et al. [11], when they fabricated sensors, powered by piezoelectric NGs. The concept of selfpowered devices is harvesting of mechanical energy from ambient sources to power electrical devices, without the need for batteries. Since then, numerous groups reported different selfpowered devices, including self-powered PH sensors [11,20] and self-powered UV-detectors [21]. Kim et al. have shown that external surface charges on $\mathrm{ZnO}$ NWs can influence on the piezoelectric behavior of the $\mathrm{ZnO} \mathrm{NWs}$ and nanotubes [22]. Regarding this, Xue et al. reported the application of NGs based on $\mathrm{ZnO}$ nanowires, as a self-powered active gas sensor, and other groups have pursued similar studies on $\mathrm{ZnO}$ nanowires/nano-arrays [23-25].

On the other hand, 2D semiconducting nanomaterials such as nanosheets, nanoplates, and nanowalls, have attracted much attention due to their novel and attractive physical/chemical properties including nanometer scale thickness, high surface-to-volume ratio, and high mechanical durability [26]. In spite of these interesting advantages, there are few reports on 2D nanostructure-based devices, especially in the field of piezoelectric NGs [26-28]. The other worthy point about the $\mathrm{ZnO}$ nanosheet-based $\mathrm{NGs}$, is their direct-current signal generation which is very crucial due to possibility of direct utilization of their generated power for nanodevices. However, most of the previously reported NGs generate alternating current (AC)-type output, requiring a rectification circuit, which increases the total size of the power package and dissipates power.

In this paper, combining the semiconducting/piezoelectric properties of $\mathrm{ZnO}$ nanosheets, we propose a direct-current $\mathrm{ZnO}$ nanosheet-based $\mathrm{NG}$ as an efficient self-powered gas sensor. To the best of our knowledge, there has been no report on 2D nanosheet-based direct-current self-powered gas sensors. In addition to large surface-to-volume ratio and high surface adsorption activity, high mechanical and chemical stability are among other worthy properties of $\mathrm{ZnO}$ nanosheets, which can entitle the realized NG as an efficient gas sensor. $\mathrm{ZnO}$ nanosheets are synthesized by hydrothermal method, which is simple equipment, low temperature, cost effective and catalyst-free method, and also benefits from large area uniform production and environmental friendliness. High output sensitivity and efficient direct-current energy scavenging are proved as the benefits of the proposed self-powered gas sensor which is able to actively detect various gases, without requiring external power. 


\section{Experimental section}

Fig.1.a presents the structure of the proposed NG, schematically. It can be observed in this figure that $\mathrm{ZnO}$ nanosheets have been grown on $\mathrm{Al}$ layer, which serves as the bottom ohmic contact, while the upper $\mathrm{Ni} / \mathrm{ZnO}$ contact serves as the Schottky contact. As the first step of fabrication process, a $100 \mathrm{~nm} \mathrm{Al}$ layer is deposited on a pre-cleaned soda lime glass by thermal evaporation method, at a base pressure of about $10^{-6}$ Torr. This metallic layer will serve as the bottom electrode, which is in ohmic contact with $\mathrm{ZnO}$ nanosheets. At this stage, $\mathrm{ZnO}$ seed layer is deposited on the Al layer, chemically [29]. For this purpose, the sample is immersed in a $0.005 \mathrm{M}$ solution of zinc acetate dehydrate in ethanol, then rinsed by ethanol, and annealed at a temperature of $90^{\circ} \mathrm{C}$. This coating procedure is repeated for about three times, to achieve a $\mathrm{ZnO}$ seed layer, with a thickness of about $100 \mathrm{~nm}$. The sample is finally annealed for 2 hours, at temperature of $350^{\circ} \mathrm{C}$, in order to crystallize the coated seed layer.

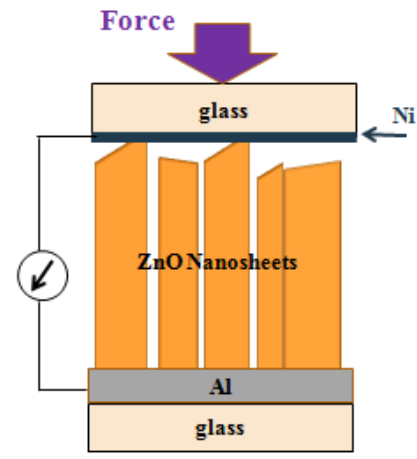

(a)

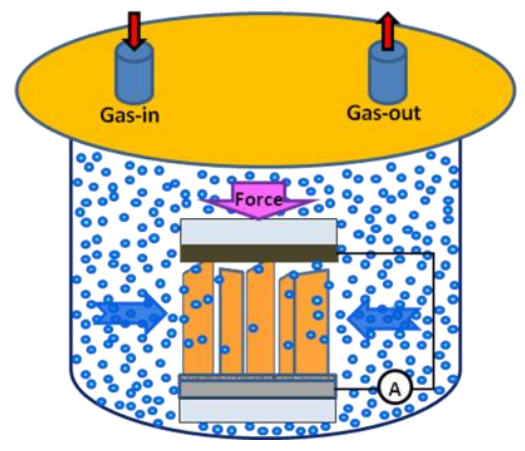

(b)

Fig. 1: (a) Structure of the fabricated NG. (b) Schematic, showing the gas sensing measurements.

Then, $\mathrm{ZnO}$ nanosheets are synthesized by hydrothermal method [26, 30-33]. At this step, a $100 \mathrm{ml}$ aqueous solution composed of zinc nitrate $\left(\mathrm{Zn}\left(\mathrm{NO}_{3}\right)_{2}-6 \mathrm{H}_{2} \mathrm{O}, 99 \%\right)$ and hexamethylenetetramine (HMT) $\left(\mathrm{C}_{6} \mathrm{H}_{12} \mathrm{~N}_{4}, 99 \%\right)$, is used as a precursor for the growth of $\mathrm{ZnO}$ nanosheets. The concentration of $\mathrm{Zn}\left(\mathrm{NO}_{3}\right)_{2}-6 \mathrm{H}_{2} \mathrm{O}$ is $20 \mathrm{mM}$, while the molar ratio of $\mathrm{Zn}\left(\mathrm{NO}_{3}\right)_{2}-6 \mathrm{H}_{2} \mathrm{O}$ to HMT is $1: 1$. The solution is then transferred into a capped Pyrex vessel in which the pre-coated substrate is floated in the reagent solution, directly beneath the cap. The resulting solution was stirred for 3 hours at a base temperature of about $90^{\circ}$, by using a magnetic stirrer. The fabricated samples were then washed in de-ionized water and air-dried. It is worth mentioning that the described chemical method leads to different $\mathrm{ZnO}$ nanostructures in the same growth conditions, but on different underlying layers. For instance, $\mathrm{ZnO}$ nanorods grow on pristine glass, while nanosheets grow exclusively on $\mathrm{Al}$ 
layer. Regarding this, formation of $\mathrm{ZnO}$ nanosheets in this process can be attributed to the underlying Al layer. Recently, a selective patterned growth of $\mathrm{ZnO}$ nanowires/nanosheets has been reported, in a single hydrothermal bath, by patterning the underlying Al layer [32]. The $\mathrm{ZnO}$ growth process, for both nanosheets and nanorods, is shown by the chemical reactions listed as follows [33]:

$\left(\mathrm{CH}_{2}\right)_{6} \mathrm{~N}_{4}+6 \mathrm{H}_{2} \mathrm{O} \rightarrow 4 \mathrm{NH}_{3}+6 \mathrm{HCHO}$

$\mathrm{NH}_{3}+\mathrm{H}_{2} \mathrm{O} \rightarrow \mathrm{NH}_{4}^{+}+\mathrm{OH}^{-}$

$\mathrm{Zn}^{2+}+2 \mathrm{OH}^{-} \rightarrow \mathrm{Zn}(\mathrm{OH})_{2}$

$\mathrm{Zn}(\mathrm{OH})_{2} \rightarrow \mathrm{ZnO}+\mathrm{H}_{2} \mathrm{O}$

It is well known that the fastest growth rate of $\mathrm{ZnO}$ is along the c-axis due to the lowest surface energy. Hence, $\mathrm{ZnO}$ nanorods are the frequent nanostructures which are achieved by hydrothermal method. However, when an underlying Al layer is present, it is believed that $\mathrm{OH}^{-}$ions are adsorbed to create $\mathrm{Al}(\mathrm{OH})_{4}{ }^{-}$, which adheres to $\mathrm{Zn}^{2+}$ terminated polar faces and suppresses growth along the c-axis. This mechanism is believed to be the main reason for lateral growth of $\mathrm{ZnO}$ on $\mathrm{Al}$ layer, and achieving 2D nanosheets instead of 1D nanorods [33]. To complete the structure of the proposed NG, a 100nm Ni layer is also deposited on another substrate by thermal evaporation. The prepared Ni-coated glass will be fixed opposite to the synthesized $\mathrm{ZnO}$ nanosheets, so that the Ni layer serve as the upper Schottky contact to the $\mathrm{ZnO}$ nanostructures (Fig. 1.a).

\section{RESUlTS AND DisCUSSION}

\section{(A) Synthesis of $\mathrm{ZnO}$ nanosheets}

Here, we study some of the effective parameters in the applied hydrothermal method for realization of $\mathrm{ZnO}$ nanosheets. As the first step, we have changed the thickness of Al layer. Fig. 2.a-c display the SEM results of hydrothermal growth on different thicknesses of $\mathrm{Al}$ layer on glass. It is observable that by decreasing the thickness of underlying $\mathrm{Al}$ layer, $\mathrm{ZnO}$ structures change from nanosheets to nanorods, which confirms the crucial role of Al layer for formation of nanosheets. Next, we have changed the concentration of zinc-acetates on the same thickness of Al layer $(100 \mathrm{~nm})$. Fig. 2.d-f present the resulted SEM images, which reveal higher sheet density and smaller lateral size for higher concentration of zinc-acetates. It can be observed in these images, that the grown nanosheet array consists of a networked structure of aligned $\mathrm{ZnO}$ nanosheets, with a thickness of about $50 \mathrm{~nm}$ and average lateral size of about $2-5 \mu \mathrm{m}$. It is notable that in the rest of this manuscript, $\mathrm{ZnO}$ nanosheets with zinc- 
acetates concentration of about $20 \mathrm{mM}$ (Fig. 2.d) have been utilized, for more investigation and fabricating piezoelectric NG.

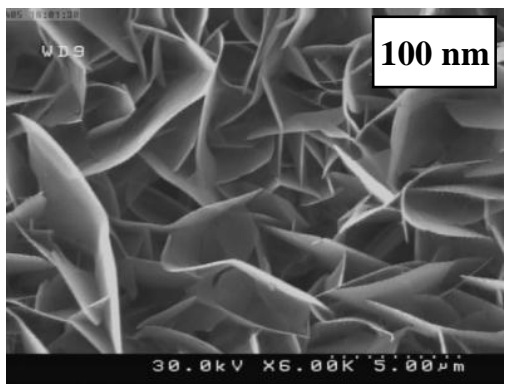

(a)

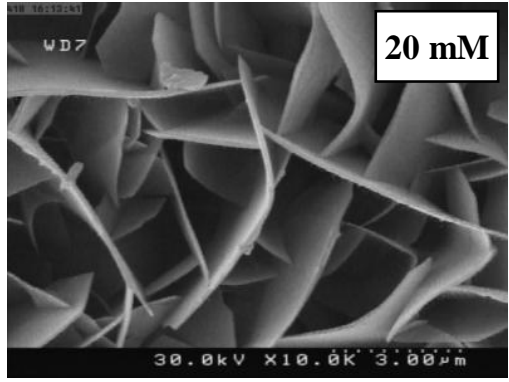

(d)

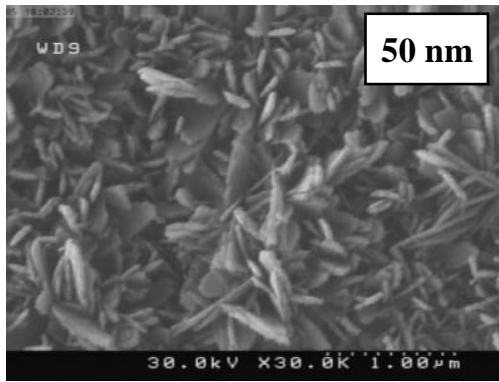

(b)



(e)

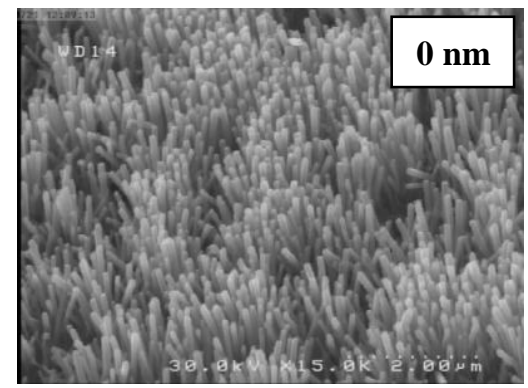

(c)

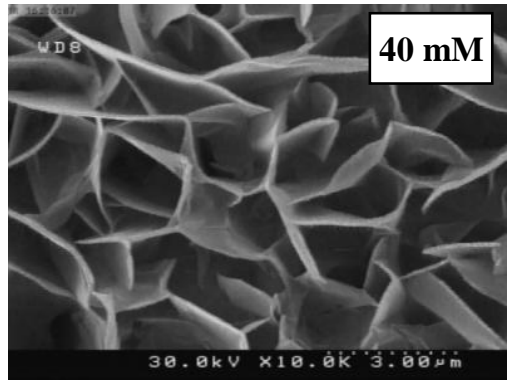

(f)

Fig. 2. The results of hydrothermal growth on different thickness of Al layer on glass: (a) $100 \mathrm{~nm}$, (b) $50 \mathrm{~nm}$, and (c) $0 \mathrm{~nm}$. SEM images of the grown nanosheets with different concentrations of zinc-acetates: (d) $20 \mathrm{mM}$, (e) $30 \mathrm{mM}$, (f) $40 \mathrm{mM}$.

To elaborate more on the morphology of the grown nanosheets, we present the lateral size distribution of the grown nanosheets (relating to Fig. 2.d) in Fig. 3.a. This plot confirms an average lateral size of about $5 \mu \mathrm{m}$ for the grown nanosheet. In addition, we present the height distribution and surface roughness of the nanosheets, achieved from AFM, in Fig. 3.b and 3.c respectively. Part (b) shows an average height of about $2.2 \mu \mathrm{m}$ for the nanosheets, which is in accordance with the presented surface roughness in part (c). To study the structure of the grown nanosheets, we have also prepared the XRD spectrum of the sample. Fig. 3.d indicates the XRD analysis of the prepared samples. Diffraction peaks originating from the hexagonal $\mathrm{ZnO}(002)$ and (101) planes are observable at $34.5^{\circ}$ and $39.5^{\circ}$. Also, three additional peaks, at around $18.5^{\circ}, 34^{\circ}, 60.1^{\circ}$ are attributed to (006), (012), and (110) crystalline planes of $\mathrm{Zn}: \mathrm{Al}$ mixed oxide layer, present at the nanosheet/Al interface. This evidence is in accordance with previous similar reports in which micro-Raman spectroscopy and XRD analysis were utilized to confirm the existence of a layered double hydroxide (LDH) or ZnAl:mixed oxide layer at the interface between the $\mathrm{ZnO}$ nanosheets and $\mathrm{Al}$ electrode [26]. 


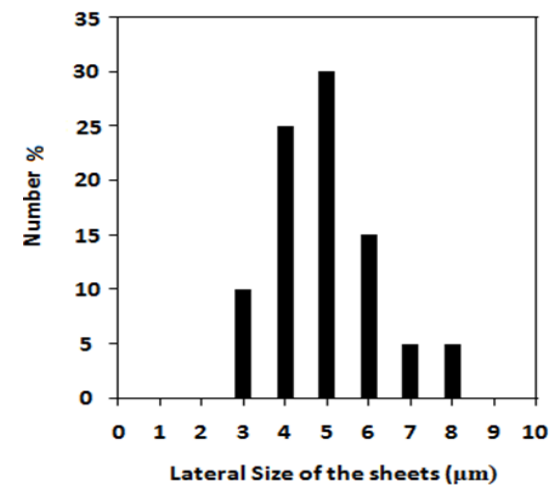

(a)

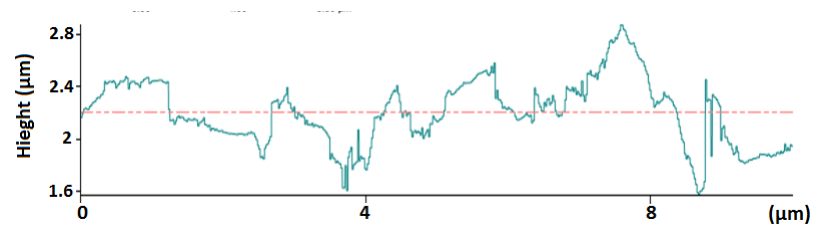

(c)

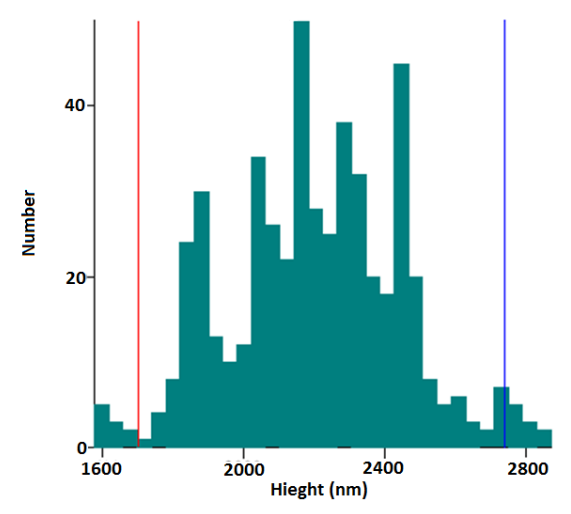

(b)

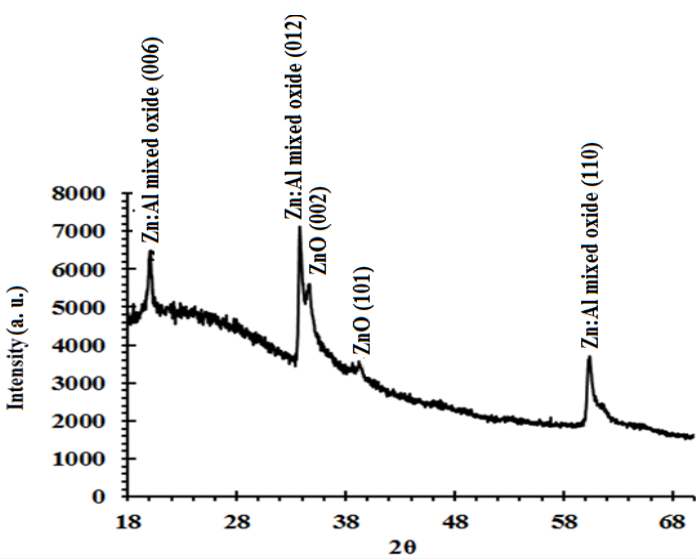

(d)

Fig. 3: (a) lateral size distribution, (b) height distribution, and (c) surface roughness of the of the grown nanosheets. (d) XRD spectrum of the synthesized $\mathrm{ZnO}$ nanosheets.

\section{(B) Piezoelectric NG based on $\mathrm{ZnO}$ nanosheets}

To elaborate the electrical characteristic of the fabricated NG, we have first investigated I-V characteristics of the relaxed structure. It is well established that a Schottky (ohmic) contact is formed at the interface of $\mathrm{Ni}(\mathrm{Al})$ with $\mathrm{ZnO}$, due to the high (low) workfunction of $\mathrm{Ni}(\mathrm{Al})$. Fig. 4.a shows the I-V characteristic of the unloaded $\mathrm{Ni} / \mathrm{ZnO} / \mathrm{Al}$ structure (black curve) in the inset, which shows a rectifying behavior attributed to the $\mathrm{Ni} / \mathrm{ZnO}$ Schottky contact. At the next stage, the stress induced variations of the electrical characteristics have been studied. I-V characteristics of the stressed structure are plotted in Fig. 4.a as red, green and blue curves, when different force values are applied. Fig. 4.c displays the band diagram of the forward biased structure, in which $\mathrm{ZnO}$ nanosheets are assumed n-type, due to the presence of native defects/impurities that can act as donors [34-37]. When an external stress is applied to $\mathrm{ZnO}$ nanosheets, a built-in field is induced in the $\mathrm{ZnO}$ nanosheet so that the compressed side becomes negative and the stretched side becomes positive (Fig. 4.c). Hence, the corresponding potential barrier and the electrical characteristic are affected by the applied 
stress. Regarding this, it is expected that the larger the applied force, the larger bias voltage would be needed to flow current or equally, a smaller current is measured. This result is in agreement with the measured data presented in Fig. 4.a.

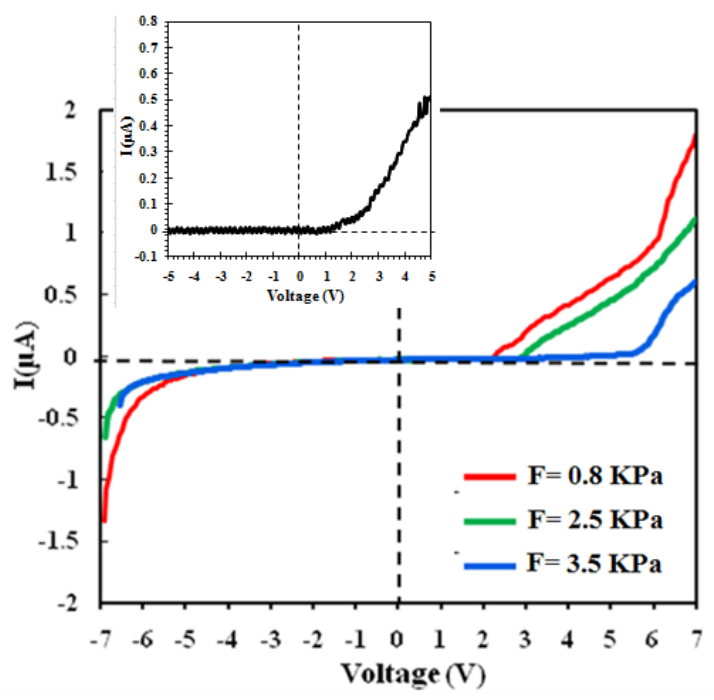

(a)

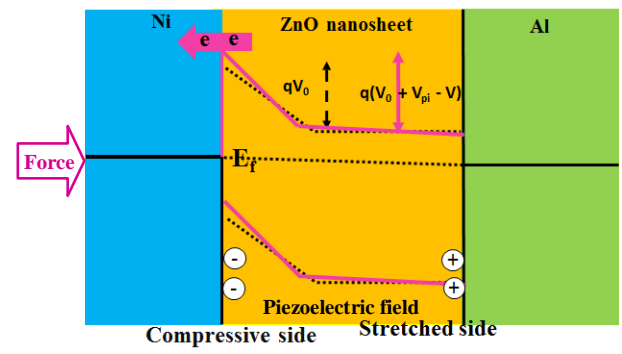

(c)

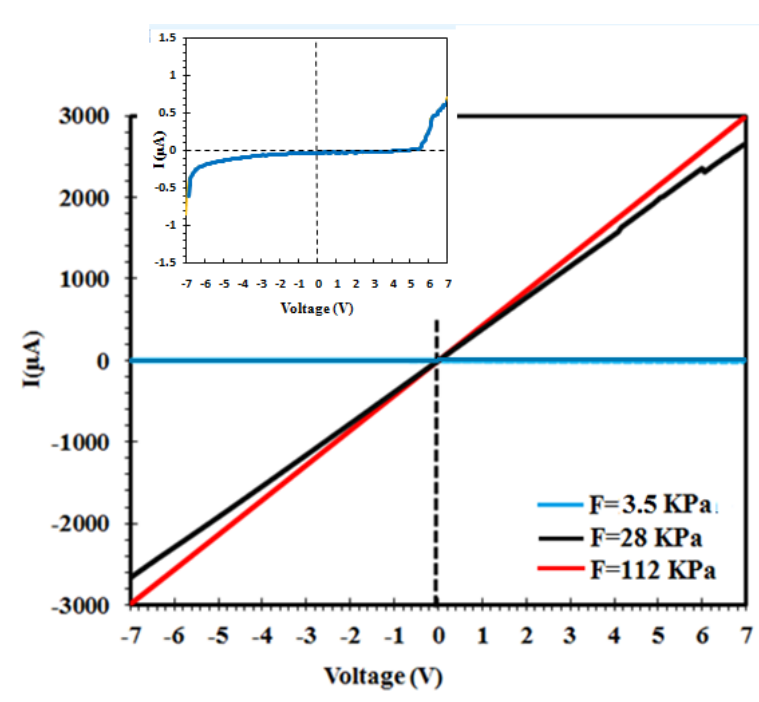

(b)

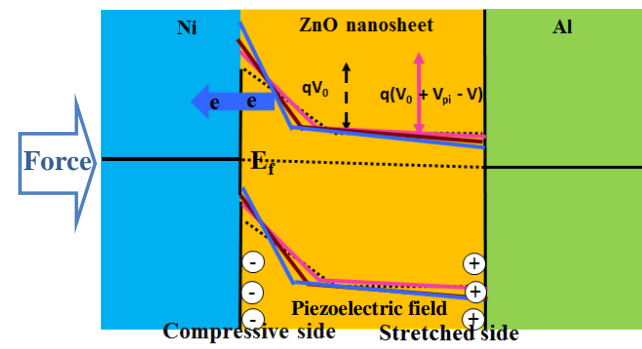

(d)

Fig. 4. (a) I-V characteristics of the fabricated NG, for different applied force values, which shows a rectifying behavior. Inset shows the rectifying characteristic of the relaxed structure, corresponding to the $\mathrm{ZnO} / \mathrm{Ni}$ Schottky contact. (b) The electrical characteristics at high level applied pressures (red and black curves), which show a linear characteristic in comparison with the case of lower loads (blue curve). Inset magnifies the currentvoltage behavior of the NG, with external pressure of $3.5 \mathrm{KPa}$. (c) The energy diagram of the forward biased NG, when an external force is applied. (d) Excessive force reduces the width of the potential barrier and can lead to dominance of tunneling current.

To clarify the effect of pressure value, the I-V characteristics of the NG are plotted for larger forces (red and black curves), superimposed on the characteristic of a smaller force (blue curve) in Fig. 4.b. It can be observed that larger force values lead to a linear electric behavior, in comparison with the rectifying behavior of the lower forces. Inset indicates a magnified plot of the blue curve. This observation can be explained by the band diagram in Fig. 4.d. It can be observed in Fig. 4.d that excessive force and high induced piezopotential can lead to narrower barriers, so that tunneling current becomes significant and the junction can show ohmic behavior. 
The fabricated NG can lead to an open circuit voltage $\left(\mathrm{V}_{\mathrm{oc}}\right) /$ short circuit current $\left(\mathrm{I}_{\mathrm{sc}}\right)$, when exposed to an external force. Fig. 5.a presents the measured time resolved $\mathrm{I}_{\mathrm{sc}}$ (blue curve), superimposed on the $\mathrm{V}_{\mathrm{oc}}$ (red curve) of the fabricated NG, when exposed to $300 \mathrm{~Pa}$ pressure. It can be observed in this figure that $\mathrm{I}_{\mathrm{sc}}$ increases to more than $140 \mathrm{nA}$ by applying force and drops down to zero, again. However, it is observable that $\mathrm{V}_{\text {oc }}$ increases to about $1.7 \mathrm{~V}$ and remains constant, until the external force is present. The other worthy point about the measured $I_{s c}$ is presented in the inset of Fig. 5.a, which shows a direct-current output as a result of applying three pressing/releasing cycles to the NG. The observed direct-current output of the $\mathrm{ZnO}$ nanosheet-based $\mathrm{NG}$, has been reported by Kim et al., for the first time [26]. They attributed the direct-current behavior to existence of a layered double hydroxide (LDH) or $\mathrm{Zn}$ and $\mathrm{Al}$ mixed oxide, at the interface between the $\mathrm{ZnO}$ nanosheets and the $\mathrm{Al}$ electrode. Our XRD spectrum also proves the existence of $\mathrm{Zn}$ and $\mathrm{Al}$ mixed layer in the synthesized nanosheet sample (Fig. 3.d). LDH behaves as an anion exchanger, and a capacitor with weak conductivity, which is believed to be responsible for the observed DC output from the realized NG [26,28]. When an external force is applied to the NG, the induced piezoelectric potential behaves as a voltage applied for storing the charges, from the nanosheets in the capacitor-like LDH layer. Hence charges transported from the nanosheets are stored into the LDH layer under an applied external force, and an output current signal is generated consequently. When the force is released, the generated piezopotential disappears, and the weak conductivity of the LDH can allow internal discharging of this capacitor. Regarding the latter discussion, Fig. 5.b displays the operation mechanism of the NG schematically. Part b-1 in this figure indicates the relaxed NG, with the ZnAl:LDH layer at the interface of the $\mathrm{ZnO}$ nanosheets and the $\mathrm{Al}$ electrode. Pressing the top electrode leads to a piezopotential along the nanosheets, with a negative polarity at the top end and a positive polarity at the bottom end (Fig. 5.b-2). Thus, electrons are drifted and transferred from the bottom contact to the $\mathrm{LDH}$ layer, compensate the positive charges in $\mathrm{LDH}$, and a net negative charge is built up in the LDH, consequently (Fig. 5.b-3). This negative charge drives electrons from Al to Ni electrode and a current signal is generated, according to Fig. 5.b-3. When the external force is released, the generated piezopotential disappears, and the negative charge of LDH layer prevents the reverse flow of electrons. The accumulated electrons are injected from the top electrode to the $\mathrm{ZnO}$ nanosheets, and the charged weakly conductive capacitor (LDH) discharges internally. 


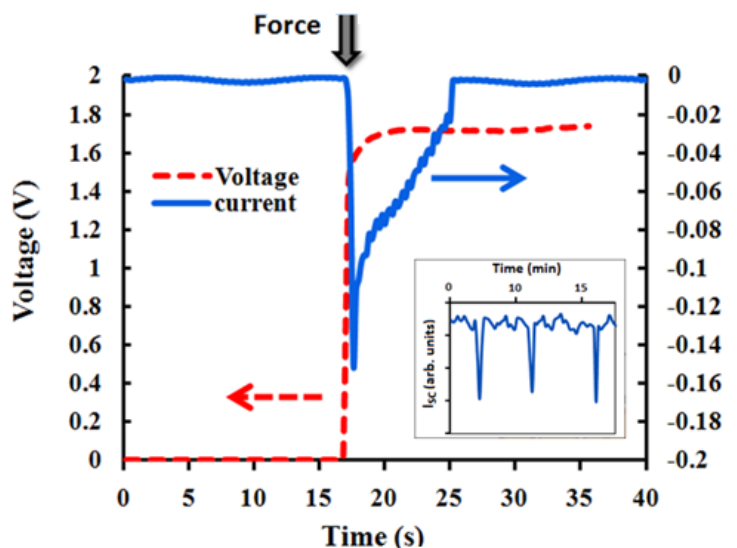

(a)
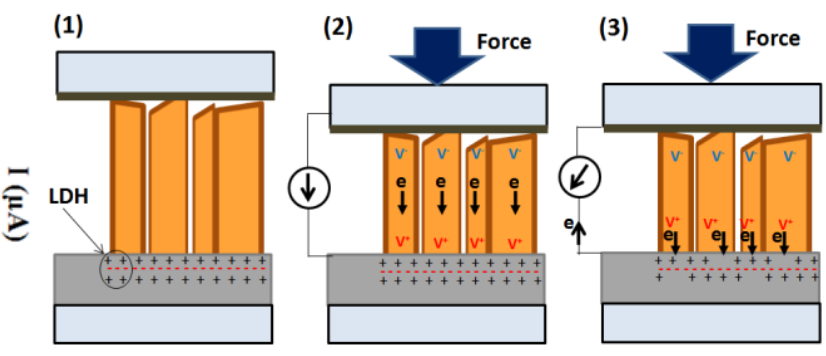

Fig. 5. (a) Time resolved response of the NG, with a pressure of about $300 \mathrm{~Pa} . \mathrm{I}_{\mathrm{sc}}$ is demonstrated by blue curve and $\mathrm{V}_{\mathrm{oc}}$ is displayed by red curve. The inset presents the measured $\mathrm{I}_{\mathrm{sc}}$ as a consequence of multiple pressing/releasing cycles, which reveals a direct-current operation for the realized nanosheet-based NG. (b) Operation mechanism of the fabricated NG. b-1) The relaxed NG, with the ZnAl:LDH layer at the interface of the $\mathrm{ZnO}$ and the $\mathrm{Al}$ electrode. $\mathrm{b}-2$ ) External force results in generating piezopotential across the nanosheets, which leads to drift of electrons. b-3) Free electrons are transferred to the weakly conductive LDH layer, which causes a net negative charge in the $\mathrm{Al} / \mathrm{ZnO}$ interface and an external current signal, consequently.

It is worth mentioning that in the field of piezoelectric NGs, there are numerous reports on structures with both flat $[4,23,26]$ and rough top electrode $[3,6]$. However, it should be noted that the morphology, roughness and the electrical properties of the top electrode can affect the characteristics of the NGs. In this regard, Choi et al. investigated the effect of nano-scale roughness of the top electrode on the performance of a piezoelectric $\mathrm{NG}$ based on $\mathrm{ZnO}$ nanorods [5]. To elaborate the effect of surface roughness of the top electrode on the current scavenging performance, we prepared a different sample based on a rough top electrode and compared the output results. For this purpose, we first synthesized $\mathrm{ZnO}$ nanorods directly on glass substrate, by a hydrothermal method similar to the aforementioned process. Time duration of the growth step was decreased to about 1 hour and the resulted nanorods had a diameter of about $100 \mathrm{~nm}$ and a length of about $300 \mathrm{~nm}$. Then we have deposited $\mathrm{Ni}$ on the prepared sample, to achieve an electrode, with nano-scale roughness. Fig. 6.b manifests schematically that applying a rough top electrode increases the number of nanosheets contacting with Ni layer $\left(\mathrm{N}_{\mathrm{c}}\right)$, and can guarantee a more reliable metal/semiconductor contact. A simple equivalent circuit has been attributed to the structure which is illustrated in Fig. 6.b. $\mathrm{R}_{\mathrm{T}}, \mathrm{R}_{\mathrm{B}}, \mathrm{R}_{\mathrm{NS}}, \mathrm{R}_{\mathrm{TC}}$ and $\mathrm{R}_{\mathrm{BC}}$ represent the resistance of top electrode (Ni layer), bottom electrode (Al layer), $\mathrm{ZnO}$ nanosheets, the top contact and the bottom contact, respectively. Fig.6.c demonstrates the I-V characteristics of the prepared NGs, in which the blue curve 
attributes to the rough, and the red curve is related to the flat electrode. It can be observed that the current level increases in the sample with rough electrode which can be explained by the equivalent resistance. Applying a rough electrode can increase $\mathrm{N}_{\mathrm{c}}$ and reduce the total resistance of the structure, according to Equ.1. Decreased resistivity leads to an increased current level, which is in agreement with our measurement (Fig. 6.c).

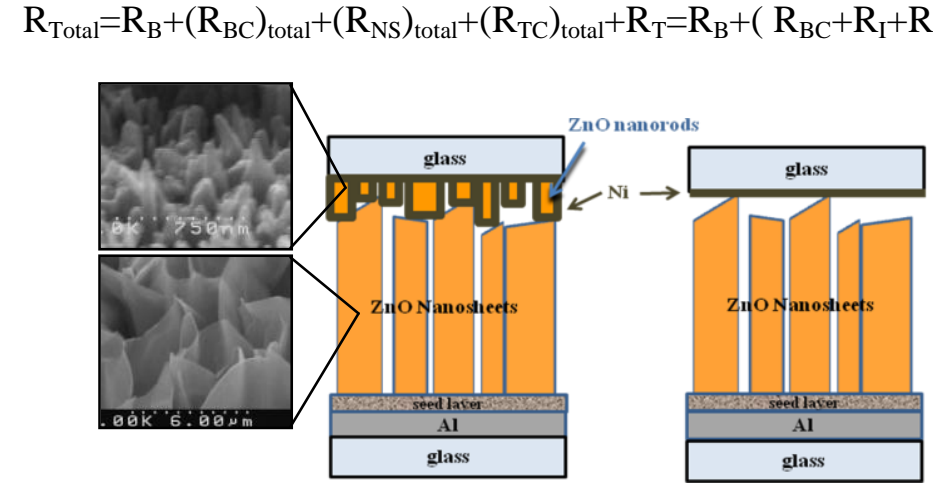

(a)

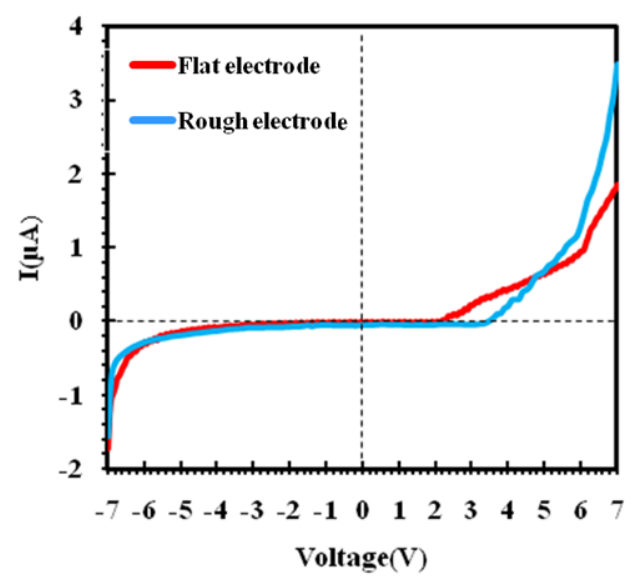

(c)

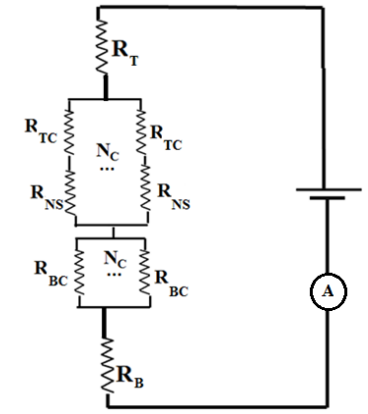

(b)

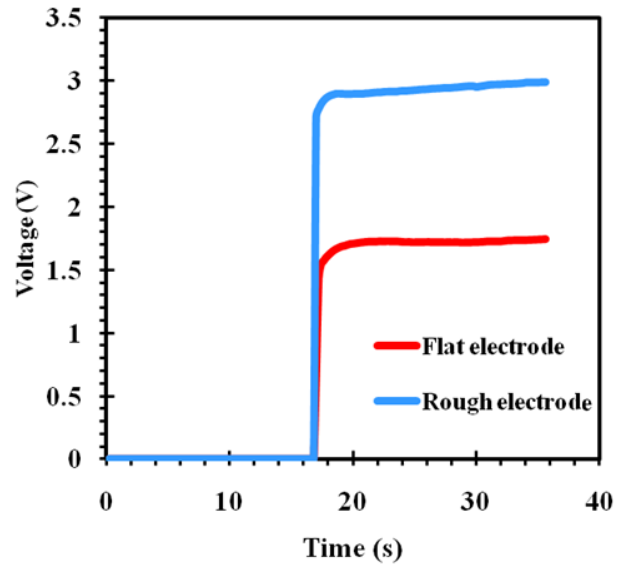

(d)

Fig. 6. (a) Schematic of $\mathrm{ZnO} / \mathrm{Ni}$ contact in the $\mathrm{NG}$, when top electrode has nano-scale roughness (left), or has a flat surface (right). (b) A simplified electric model, for the realized NG. (c) I-V characteristics of the NGs with rough (blue curve) and flat (red curve) top electrodes. (d) The time resolved open circuit output of the NGs with rough (blue) and flat (red) top electrodes.

Fig. 6.d exhibits the open circuit output of the fabricated NGs, in which $\mathrm{V}_{\mathrm{oc}}$ is increased when a rough top electrode is utilized. This observation can also be related to better mechanical coupling between the nanosheets and the top electrode, which results in better mechanicalelectrical conversion in the NG. It should be noted that the described NGs were investigated under the same external pressure, in this experiment. 


\section{(C) Gas sensing operation of the $\mathrm{ZnO}$ nanosheet-based NG}

It is known that free carriers in the piezoelectric semiconductors can drift in a direction opposite to the induced piezoelectric field and screen the induced $\mathrm{V}_{\mathrm{oc}}$, consequently. In this regard, the effect of external surface charges on the piezoelectric behavior of semiconducting piezoelectric nanostructures has been demonstrated by simulation, previously [22]. This is the basic idea, which has been applied in this paper, to realize self-powered gas sensors. For this purpose, first we have studied the current-voltage characteristic of our NG, when exposed to different concentrations of humidity. Hence, we have exposed the unpackaged NG to different concentrations in a closed test chamber, and investigated the output characteristics (Fig. 1.b). Fig. 7.a indicates the variation of I-V characteristics, when NG is exposed to different humidity concentrations. An external pressure of about $300 \mathrm{~Pa}$ is applied to the NG, in this experiment.

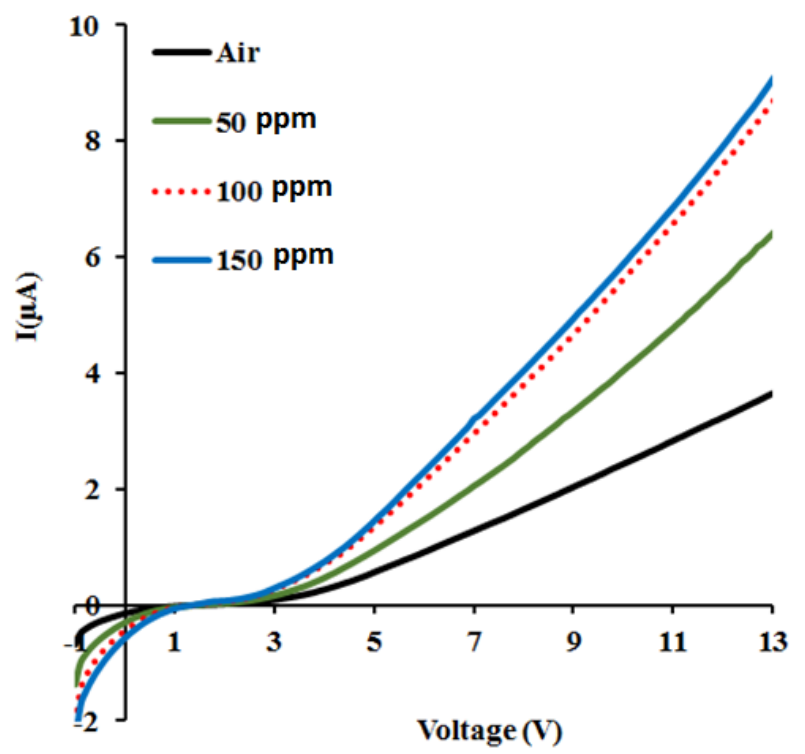

(a)

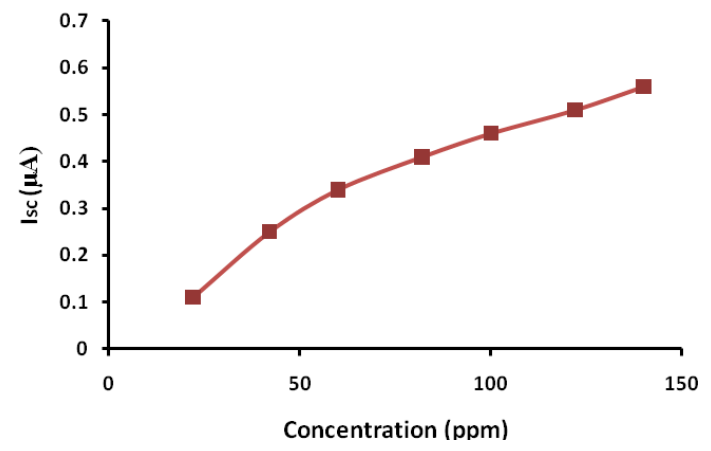

(c)

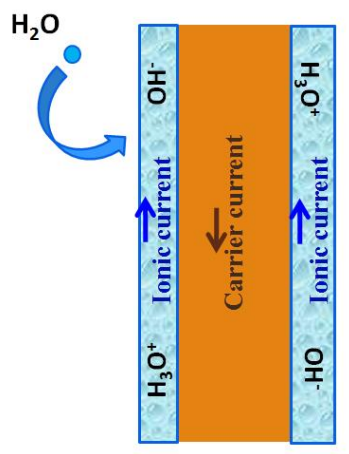

(b)

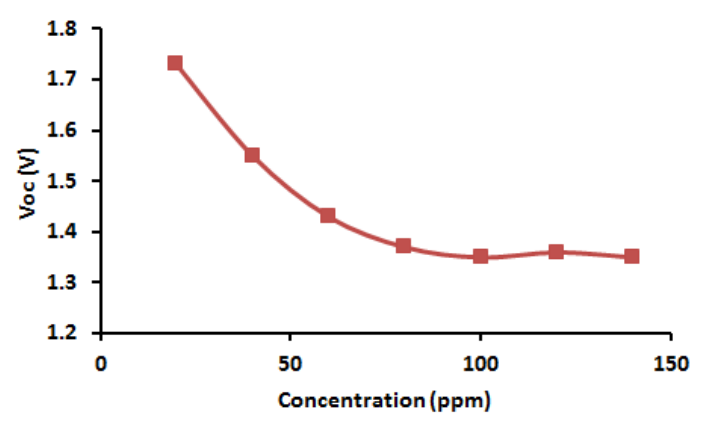

(d) 
Fig. 7. (a) The achieved I-V curves related to different concentrations of humidity. (b) Water vapor absorption on $\mathrm{ZnO}$ nanosheet. Variation of: (c) short-circuit current, and (b) open circuit voltage versus changing humidity concentration, at room temperature.

It was noted previously that the rectifying electrical behavior of NG can be affected by both the value of external pressure (Fig. 4.b), and the surface charges induced by adsorbed gas molecules. Hence, as can be observed in Fig. 7.a I-V characteristics are deviating from rectifying behavior, when exposed to external force and gas concentration, simultaneously. It is observable in this figure that higher humidity results in higher current level. When exposed to humidity, water molecules are chemisorbed on the surface of $\mathrm{ZnO}$ nanosheets and $\mathrm{OH}^{-}$and $\mathrm{H}_{3} \mathrm{O}^{+}$groups are formed at the surface of nanosheets (Fig. 7.b). Subsequent water molecules are physisorbed on the initially adsorbed monolayer and a water layer is formed on the surface of nanosheets. Charge transfer can occur in the surface water layer by releasing $\mathrm{H}_{3} \mathrm{O}^{+}$ ion from neighboring water molecules to next molecules. So $\mathrm{H}_{3} \mathrm{O}^{+}$ions can be considered as charge carriers in addition to free electrons, as depicted schematically in Fig. 7.b [23].

To clarify the effect of humidity on the performance of $N G, I_{s c}$ and $V_{o c}$ have been measured and plotted versus humidity concentration, in figures 7.c and 7.d, respectively. It is shown that the higher concentration of humidity, the higher $\mathrm{I}_{\mathrm{sc}}$ and the lower $\mathrm{V}_{\mathrm{oc}}$ are resulted. Under compressive stress, both $\mathrm{H}_{3} \mathrm{O}^{+}$in the physisorbed water layer, and the free electrons inside the $\mathrm{ZnO}$ nanosheets can drift to screen the piezoelectric potential and decrease $\mathrm{V}_{\mathrm{oc}}$ value. Also, addition of mobile $\mathrm{H}_{3} \mathrm{O}^{+}$to charge carriers can lead to a shunt parasitic conductive layer and a higher $I_{s c}$, consequently. The short circuit sensitivity $\left(\mathrm{S}_{\mathrm{sc}}=\frac{I_{s c 2-I_{s c 1}}}{I_{s c 1}}\right)$ and the open circuit sensitivity $\left(S_{o c}=\frac{V_{o c 2}-V_{o c 1}}{V_{o c 1}}\right)$ of the realized $\mathrm{NG}$, are achieved about $43.4 \%$ and $-2.96 \%$, in response to increasing the humidity concentration for about $10 \mathrm{ppm}$. It is also notable that the observed nonlinearities in the output characteristics (parts c ,d) can be attributed to initializing surface absorption saturation of gas molecules on the nanosheets, which is generally responsible for saturation of output behavior in gas sensors, at high concentrations. On the other hand, non-uniformities in the synthesized nanosheets can also play role in the observed nonlinearities.

Comparing this work with recent publications shows that the achieved short circuit sensitivity $\left(\sim 58.4\left(\frac{\Delta I_{s c}}{I_{S c}}\right)\right.$ versus $1 \%$ increasing of $\left.\mathrm{RH}\right)$ is more than 10 times higher than the reported maximum open circuit sensitivity of a nanorod-based self-powered humidity sensor $(\sim 5.5$ $\left(\frac{\Delta v_{o c}}{v_{o c}}\right)$ versus $1 \%$ increasing of $\left.\mathrm{RH}\right)[25]$. The measured output power of the NG has also 
shown about 4-fold enhancement (increased from $47 \mathrm{nW} / \mathrm{cm}^{2}$ to $192 \mathrm{nW} / \mathrm{cm}^{2}$ ), when exposed to a maximum humidity concentration of about $150 \mathrm{ppm}$. Moreover, according to the latter results (Fig. 7) and discussions, humidity can alter $\mathrm{I}_{\mathrm{sc}}$ and $\mathrm{V}_{\mathrm{oc}}$ of the $\mathrm{NG}$, individually. Hence, two separate output parameters are accessible to monitor, as the fingerprint of humidity. This observation can be utilized to realize humidity sensors, which are taking advantage of high sensitivity, better selectivity, beside a self-powered operation, in comparison with the conventional resistive sensors.

We have also, studied the time resolved response of the realized self-powered gas sensor to humidity. For this purpose, the output behavior of the sensor was monitored after a sudden exposure to a constant concentration of humidity. Fig. 8.a depicts a rise time of about $11 \mathrm{~min}$, which is related to the long time it takes for water molecules to diffuse into the unpackaged NG and approach to the inner nanosheets. During this time gas molecules have the chance to diffuse through the sidewalls of NG, approach/adsorb on more nanosheets, and influence the output of the NG, more effectively. This point is the main reason that causes a slow gas sensing response in the proposed package, despite of a fast piezoelectric response of the NG to external stress. The penetration of gas molecules is highlighted in the inset schematically, in which blue arrows illustrate the diffusion directions. At the next step, the gas-in valve was closed and the decreasing behavior of $\mathrm{I}_{\mathrm{sc}}$ was monitored during time. Figure 8.b indicates a recovery time of about $25 \mathrm{~min}$, for the fabricated sensor. Slow desorption of water molecules from the surface of $\mathrm{ZnO}$ nanosheets is another reason for the observed long recovery time.

To improve the response time of the proposed self-powered gas sensor, we have utilized a micro-scale porous electrode as the top electrode. The proposed structure is presented schematically in the inset of Fig. 8.c, in which blue arrows depict diffusion directions of gas molecules. Top electrode in this structure is a PET which is Ni-coated, and perforated to realize a hole array with diameter and spacing of about $400 \mu \mathrm{m}$ and $2 \mathrm{~mm}$, respectively. The proposed structure provides shorter diffusion path for gas molecules, and all nanosheets have the chance to be exposed to gas molecules simultaneously. Fig. 8.c indicates the response of the modified device, which reveals a rise time of about $70 \mathrm{Sec}$, attributed to shorter diffusion path for gas molecules. Also, output sensitivity is enhanced by a factor of about 2.3, which is related to parallel exposure of more nanosheets to humidity. Hence, the presented sensor shows a response time, comparable to the typical humidity sensors, and benefiting from selfpowered functionality. 


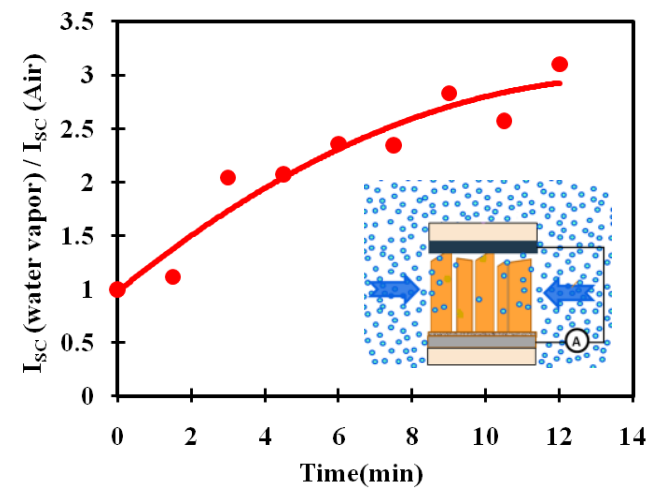

(a)

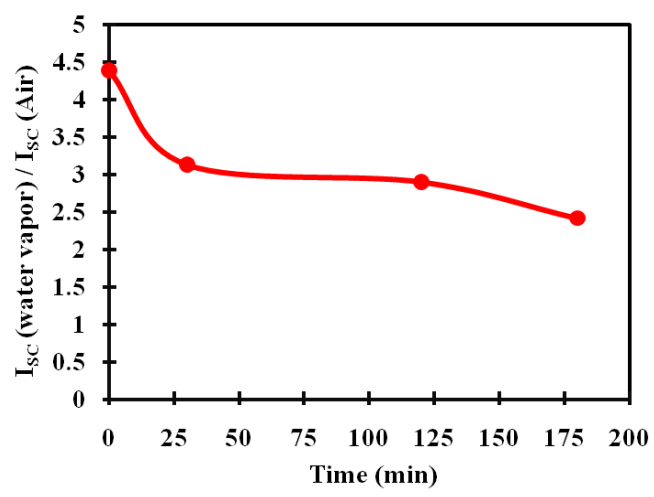

(b)

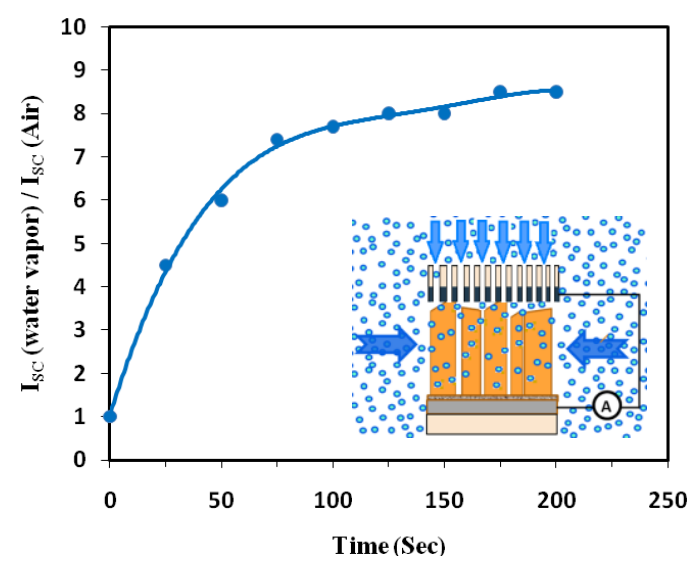

(c)

Fig. 8. Time resolved variation of the measured $I_{s c}$, relative to the initial value; (a) when gas-in valve is opened at $\mathrm{t}=0$; (b) when gas-in valve is closed. A rise time of about $11 \mathrm{~min}$ and a recovery time of about 25 min are observed for the realized self-powered humidity sensor. (c) The measured fast response of the nanosheet based sensor, when a micro-porous electrode is utilized as the top electrode. An enhanced rise time of about $70 \mathrm{sec}$ and 2.3-fold enhancement in sensitivity, are achieved in the modified structure. Insets illustrate the allowable diffusion directions for gas molecules, in the relating structures.

To elaborate the dynamic response of the realized self-powered gas sensor, we have prepared another sample and studied the time resolved output of the NG, in response to different gas-in concentrations. Fig. 9 exhibits the time variations of $\mathrm{I}_{\mathrm{sc}}$, while humidity concentrations has been varied. Red/blue arrows show the pressing/releasing time incidents in this figure. The applied external pressure was about $300 \mathrm{KPa}$, in this experiment. It is notable that there has been adequate time intervals between exposure of different gas concentrations in this experiment, to assure realizing a steady state condition for the fabricated gas sensor. It can be observed that increasing concentration from 20 ppm to $150 \mathrm{ppm}$, leads to increasing $\mathrm{I}_{\mathrm{sc}}$ from about 0.1 to $0.6 \mu \mathrm{A}$, which is in accordance with (Fig. 7.c). The other worthy pint about this figure is the observed acceptable 
repeatability in the output characteristics of the fabricated sensor. Our study shows an average accuracy of about $9 \%$ for the measured short circuit output $\left(\mathrm{I}_{\mathrm{sc}}\right)$.

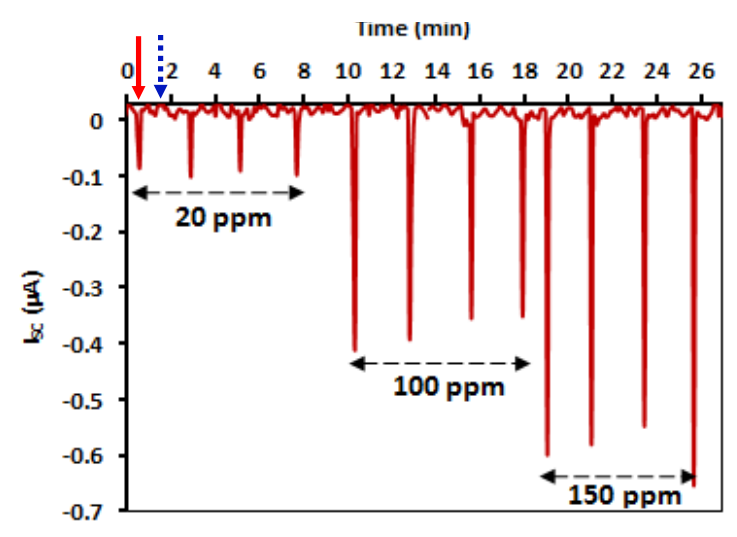

Fig. 9. Transient response of the realized self-powered gas sensor to humidity, when an external pressure of about $300 \mathrm{~Pa}$ is exerted to the NG. Red/blue arrows show the pressing/releasing time incidents. It is shown that the measured $\mathrm{I}_{\mathrm{sc}}$ is increased when humidity is introduced to the chamber.

\section{(D) Resistive operation of the $\mathrm{ZnO}$ nanosheets-based humidity sensor}

To compare the presented peizoelectric-based sensing mechanism with the conventional resistive sensing, we have investigated the resistive behavior of the nanosheets between the top and bottom electrodes. Figure 10 presents the variation of the nanosheet's resisitivity relative to their initial resistivity, when exposed to humidity. The reported resistivities are measured at a bias voltage of about $5 \mathrm{~V}$.

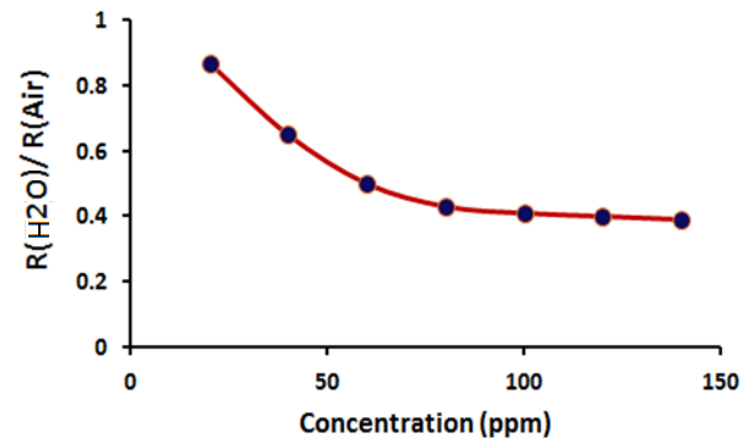

Fig. 10. Resistive operation of the relaized $\mathrm{ZnO}$ nanosheets, when exposed to humidity. Variation of the measured resistivity of the nanosheets, versus increasing concentrations of humidity.

Fig. 10 manifests that the higher humidity concentration results in lower resistivity, which is related to the shunt conductive layer of $\mathrm{H}_{3} \mathrm{O}^{+}$ions, created on the surface of nanosheets. Resistive sensitivity $(=\Delta R / R)$ of about $4.2 \%$ is measured in the resistive operation mode 
of the device, versus increasing concentration of humidity for about $10 \mathrm{ppm}$. The achieved results illustrate that the realized humidity sensor leads to about 10 times higher output sensitivity in the self-powered operation, comparing with the conventional resistive operation. Moreover, better selective detection of humidity was demonstrated in the self-powered operation, due to the proposed output variation mechanisms ( $\mathrm{I}_{\mathrm{sc}}$ and $\mathrm{V}_{\mathrm{oc}}$ ). Therefore, the presented $\mathrm{ZnO}$-nanosheet based $\mathrm{NG}$ can act not only as a self-power gas sensor, but also intoduces new prospects and enhanced output characteristics in comparison with conventional sensors.

Finally, there are obviously practical points that shoud be addressed for the proposed gas sensor, for commerciallization purposes. The cross sensitivity to other ambient parameters, such as gas flow rate and temperature, are among these points. It is believed that these cross sensitivities can be compensated by some typical techniques which are applied in the present sensing modules. For instane, the sensitivity to the mechanical force of gas flow with high flow rates, can be compensated by applying suitable packing structures like Pitot tube. Hence, it can be garanteed that the active sensing element does not sense the gas flow directly.

\section{CONCLUSIONS}

In summary, we applied hydrothermal method to synthesize $\mathrm{ZnO}$ nanosheets on $\mathrm{Al}$ layer, and utilized these mechanically strong/stable nanostructures to fabricate direct-current piezoelectric NG. Taking advantage of coupled piezoelectric/semiconducting behavior of $\mathrm{ZnO}$ nanosheets; we realized nanosheet-based self-powered humidity sensor, for the first time. The short circuit and open circuit sensitivities in response to humidity were achieved about $43.4 \%$ and $-2.96 \%$, versus increasing the humidity concentration for about $10 \mathrm{ppm}$. It is notable that the achieved short circuit sensitivity is more than 10 times higher than the previously reported open circuit sensitivity of nanorod-based self-powered humidity sensor. Therefore, it is believed that the presented $\mathrm{ZnO}$-nanosheet based NG can be entitled as a direct-current self-powered humidity sensor, with new prospects and enhanced output characteristics.

\section{Acknowledgments}


The authors would like to acknowledge the partial financial support of the Iran National Science Foundation (INSF) 92001674. Authors also wish to acknowledge Professor V. Ahmadi for his kind supports.

\section{References}

[1] Q. Wan, Q. H Li, Y. J Chen, T. H Wang, X. L He, J. P Li, C. L Lin, Fabrication and ethanol sensing characteristics of $\mathrm{ZnO}$ nanowire gas sensors, Applied Physics Letters 84(18) (2004) 3654-3656.

[2] X. Q Fu, C Wang, H. C Yu, Y. G Wang, \& T. H Wang, Fast humidity sensors based on $\mathrm{CeO} 2$ nanowires, Nanotechnology 18(14) (2007) 145503.

[3] Z. L Wang, J Song, Piezoelectric nanogenerators based on zinc oxide nanowire arrays, Science 312(5771) (2006) 242-246.

[4] S. N Cha, J. S Seo, S. M Kim, H. J Kim, Y. J Park, S. W Kim, J. M Kim, Sound-Driven Piezoelectric Nanowire-Based Nanogenerators, Advanced materials 22(42) (2010) 4726-4730.

[5] D. Choi, M. Y Choi, H. J Shin, S. M Yoon, J. S Seo, J. Y Choi, S. Y Lee, J. M Kim, S. W Kim, Nanoscale networked single-walled carbon-nanotube electrodes for transparent flexible nanogenerators, The Journal of Physical Chemistry C 114(2) (2009) 1379-1384.

[6] C. L Hsu, K. C Chen, Improving piezoelectric nanogenerator comprises $\mathrm{ZnO}$ nanowires by bending the flexible PET substrate at low vibration frequency, The Journal of physical chemistry C 116(16) (2012) 9351-9355.

[7] C. J Hu, Y. H Lin, C. W Tang, M. Y Tsai, W. K Hsu, \& H. F Kuo, ZnO-Coated Carbon Nanotubes: Flexible Piezoelectric Generators, Advanced Materials 23(26) (2011) 2941-2945.

[8] C. Y Chen, G Zhu, Y. Hu, J. W Yu, J. Song, K. Y. Cheng, L. H Peng, L. J Chou, Z. L Wang, Gallium nitride nanowire based nanogenerators and light-emitting diodes, ACSNano 6(6) (2012) 5687-5692.

[9] G. Zhu, R. Yang, S. Wang, Z. L Wang, Flexible high-output nanogenerator based on lateral ZnO nanowire array, Nano letters 10(8) (2010) 3151-3155.

[10] Y. Hu, Y. Zhang, C. Xu, G. Zhu, Z. L Wang, High-output nanogenerator by rational unipolar assembly of conical nanowires and its application for driving a small liquid crystal display, Nano Letters 10(12) (2010) 5025-5031.

[11] S. Xu, Y. Qin, C. Xu, Y. Wei, R. Yang, Z. L Wang, Self-powered nanowire devices, Nature nanotechnology 5(5) (2010) 366-373.

[12] R. Yang, Y. Qin, C. Li, G. Zhu, Z. L. Wang, Converting biomechanical into electricity by a muscle-movement-driven nanogenerator, Nano. Lett. 9(3) (2009) 1201-1205.

[13] Z. Li, G. Zhu, R. Yang, A.C. Wang, Z.L. Wang, Muscle-Driven In Vivo Nanogenerator, Adv. Mater. 22 (2010) 2534-2537.

[14] M. Lee, C.Y. Chen, S. Wang, S.N. Cha, Y.J. Park, J.M. Kim, L-J Chou, Z. L. Wang, A Hybrid Piezoelectric Structure for Wearable Nanogenerators, Adv. Mater. (2012) 00150

[15] Y. Qiu, J. Lei, D. Yang, B. Yin, H. Zhang, J. M. Bian, J. Ji, Y. Liu, Y. Zhao, Y. Luo, L. Hu, Enhanced performance of wearable piezoelectric nanogenerator 
fabricated by two-step hydrothermal process, Appl. Phys. Lett. 104(11) (2014) 113903.

[16] M. Zhang, T. Gao, J. Wang, J. L. Yingqiang, Q.Q. Yang, H. Xue, Z. Shi, Y. Zhao, Z. Xiong, L. Chen, A hybridfibers based wearable fabric piezoelectric nanogenerator for energy harvesting application, Nano Energy 13 (2015) 298-305.

[17] W.S. Jung, M.J. Lee, M.G. Kang, H. G. Moon, S.J. Yoon, S.H. Baek, C.Y. Kang, Powerful curved piezoelectric generator for wearable applications, Nano Energy 13 (2015) 174-181.

[18] X. Wang, J. Liu, J. Song, Z. L. Wang, Integrated Nanogenerators in Biofluid, Nano Lett.7(8) (2007) 2475-2479.

[19] G. T. Hwang, H. Park, J.H. Lee, S. Oh, K. Park, M. Byun, H. Park, G. Ahn, C. K. Jeong, K. No, H.S. Kwon, S.G. Lee, B. Joung, K. J. Lee, Self-Powered Cardiac Pacemaker Enabled by Flexible Single Crystalline PMN-PT Piezoelectric Energy Harvester, Adv. Mater. 26 (2014) 4880-4887.

[20] B. Saravanakumar, S. Soyoon, S. J Kim, Self-Powered pH Sensor Based on a Flexible Organic-Inorganic Hybrid Composite Nanogenerator, ACS applied materials \& interfaces 6(16) (2014)13716-13723.

[21] J. Liu, P. Fei, J. Song, X. Wang, C. Lao, R. Tummala, Z. L. Wang, S. Xu, B. J. Hansen, Z. L. Wang, Carrier Density and Schottky Barrier on the Performance of DC Nanogenerator, Nano Lett. 8(1) (2008) 328-332.

[22] S. M Kim, H Kim, Y Nam, S Kim, Effects of external surface charges on the enhanced piezoelectric potential of $\mathrm{ZnO}$ and AlN nanowires and nanotubes, AIP Advances 2(4) (2012) 042174.

[23] X. Xue, Y. Nie, B. He, L. Xing, Y. Zhang, Z. L Wang, Surface free-carrier screening effect on the output of a $\mathrm{ZnO}$ nanowire nanogenerator and its potential as a self-powered active gas sensor, Nanotechnology 24(22) (2013) 225501.

[24] W. Zang, Y. Nie, D. Zhu, P. Deng, L. Xing, X. Xue, Core-Shell In2O3/ZnO Nanoarray Nanogenerator as a Self-Powered Active Gas Sensor with High H2S Sensitivity and Selectivity at Room Temperature, J. Phys. Chem. C 118 (2014) 9209-9216.

[25] D. Zhu, T. Hu, Y. Zhao, W. Zang, L. Xing, X. Xue, High-performance selfpowered/active humidity sensing of $\mathrm{Fe}$-doped $\mathrm{ZnO}$ nanoarray nanogenerator, Sensors and Actuators B 213 (2015) 382-389.

[26] K. H Kim, B. Kumar, K. Y Lee, H. K Park, J. H Lee, H. H Lee, H Jun, D Lee, S. W Kim, Piezoelectric two-dimensional nanosheets/anionic layer heterojunction for efficient direct current power generation, Scientific reports 3 (2013) 2017.

[27] M. K. Gupta, J-H Lee, K. Y. Lee, S-W Kim, Two-dimensional Vanadiumdoped $\mathrm{ZnO}$ nanosheet-based flexible direct current nanogenerator, ACSNano 7(10) (2013) 8932-8939.

[28] G. C. Yoon, K. S. Shin, M. K. Gupta, K. Y. Lee, J. H. Lee, Z. L. Wang, S. W. Kim, High-performance hybrid cell based on an organic photovoltaic device and a direct current piezoelectric nanogenerator, Nano Energy 12 (2015) 547-555.

[29] L. E Greene, M Law, D. H Tan, M Montano, J Goldberger, G Somorjai, P Yang, General route to vertical $\mathrm{ZnO}$ nanowire arrays using textured $\mathrm{ZnO}$ seeds, Nano Letters 5(7) (2005) 1231-1236.

[30] X. Li, P. Liang, Le Wang, F. YU, Preparation and characterization of high uniformity zinc oxide nanosheets, Front. Optoelectron. 7(4) (2014) 509-512.

[31] M.K. Gupta, J.H. Lee, K.Y. Lee, S.W.Kim, Two dimensional vanadium-doped $\mathrm{ZnO}$ nanosheer-based flexible direct current nanogenerators, ACSNano 7(10) (2013) 8932-8939. 
[32] K. Chen, D. D. Thang, S. Ishii, R. P. Sugavaneshwa, T. Nagao, Selective patterned growth of $\mathrm{ZnO}$ nanowires/nanosheets and their photoluminescence properties, Optical Material Express 5(2) (2015) 353-360.

[33] J. Ding, X. Fang, R. Yang, B. Kan, X. Li, N. Yuan, Transformation of ZnO polycrystalline sheets into hexagon-like mesocrystalline $\mathrm{ZnO}$ rods (tubes) under ultrasonic vibration, Nanoscale Research Letters 9 (2014) 214.

[34] F. A Kroger, The Chemistry of Imperfect Crystals, North Holland Publishing, Amsterdam (1974).

[35] $\mathrm{S} \mathrm{Xu}, \mathrm{Z}$. L Wang, One-dimensional $\mathrm{ZnO}$ nanostructures: solution growth and functional properties, Nano Research 4(11) (2011) 1013-1098.

[36] D. C Look, , J. W Hemsky, J. R Sizelove, Residual native shallow donor in $\mathrm{ZnO}$, Physical review letters 82(12) (1999) 2552.

[37] D. G Thomas, J. J Lander, Hydrogen as a donor in zinc oxide, The Journal of Chemical Physics 25(6) (1956) 1136-1142.

\section{Biographies}

Elham Modaresinezhad received her M.Sc. degrees in 2014, from the Department of Electrical and Computer Engineering, at Tarbiat Modares University (TMU), Tehran, Iran. She has worked on $\mathrm{ZnO}$-based piezoelectric nanogenerators and their promising applications, as her master project.

Sara Darbari received her Ph.D. in Electronic Engineering from the University of Tehran, Iran, in 2011. She is now, an assistant professor in ECE department of Tarbiat Modares University (TMU), Tehran, Iran. Her research interests are electronic, optoelectronic, electromechanic and energy harvesting devices. 


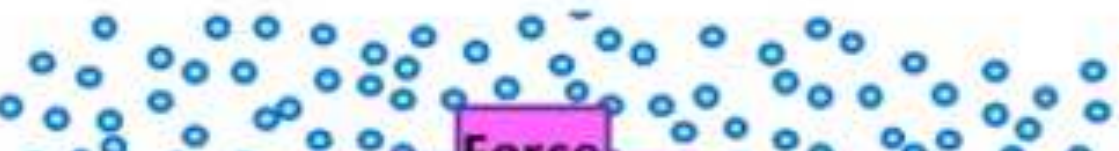
$\circ 0^{\circ} \circ 0^{\circ} \circ$ Force $0^{\circ} 0^{\circ}, 0^{\circ} \circ:$

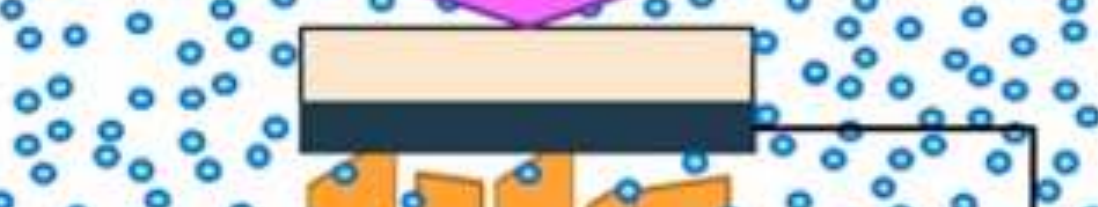

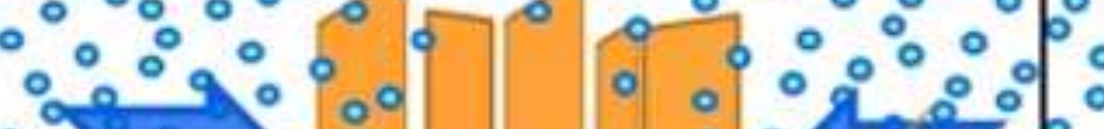
$0 \sin _{0}^{0} \%$ $08^{\circ} 0_{0}^{\circ} 0$ $\circ \circ 0^{\circ}$ ०0.0\%0 o.
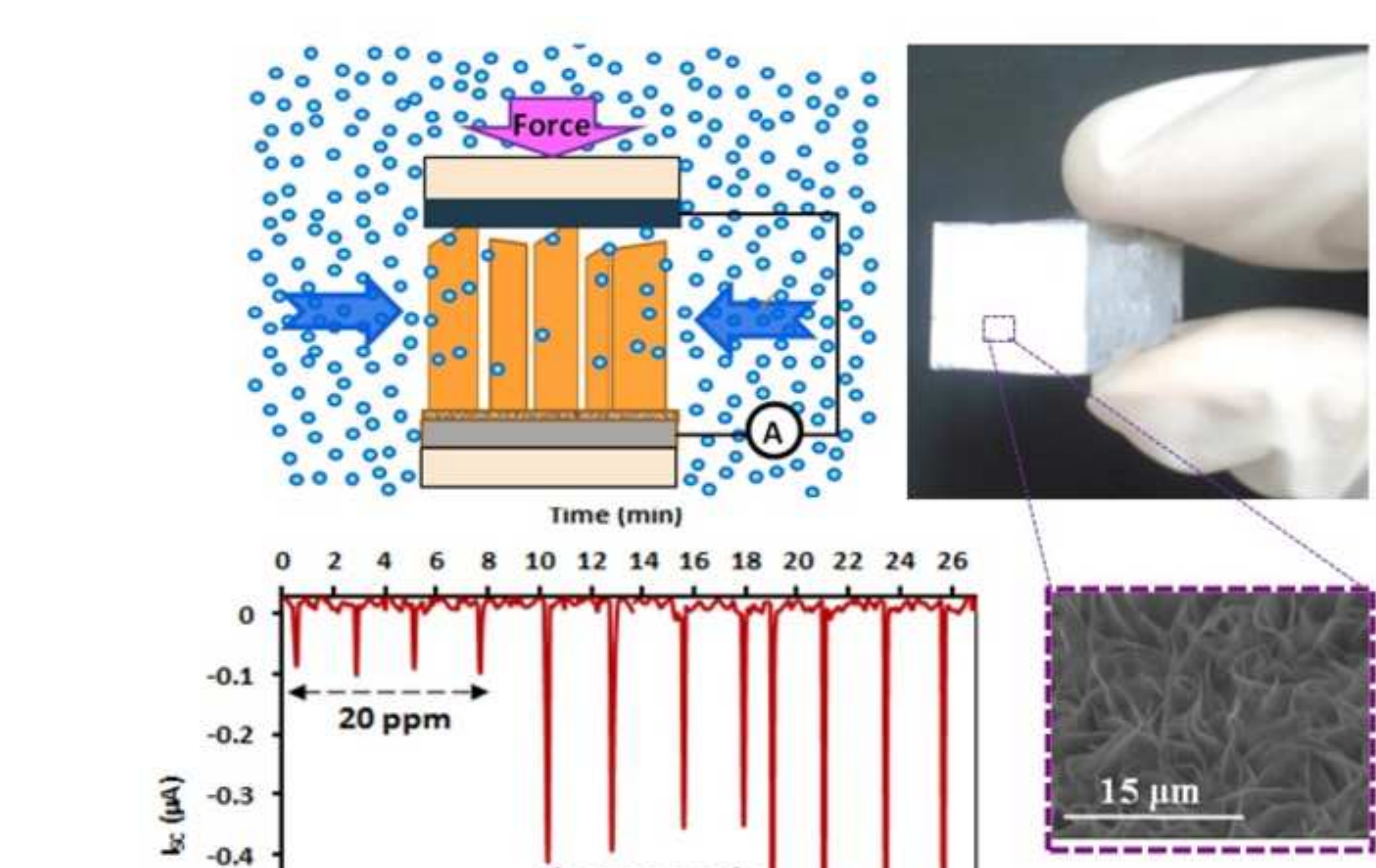

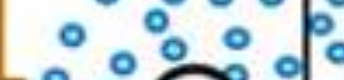

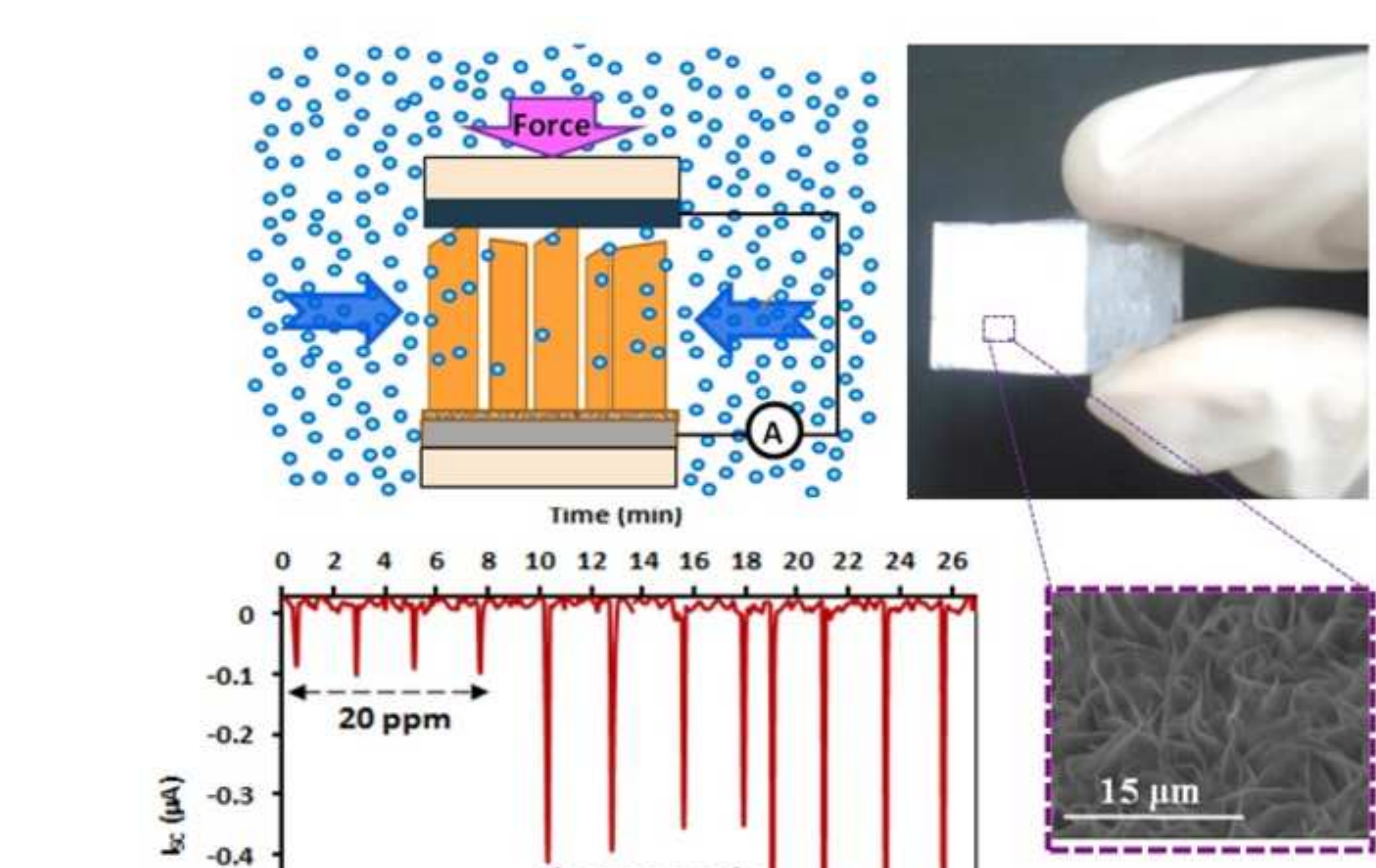
$\circ 0^{\circ} \circ \circ$

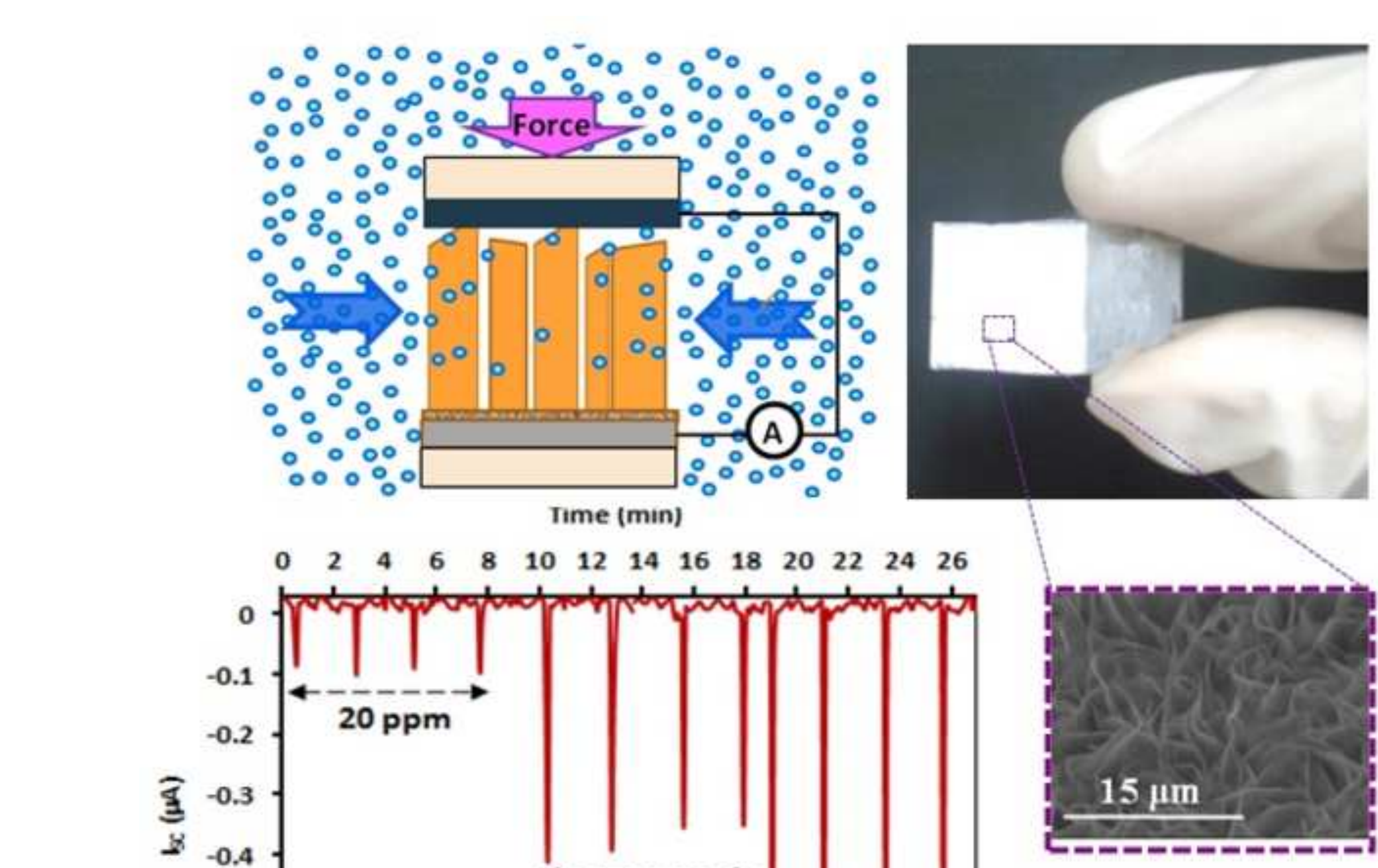
Time (min)
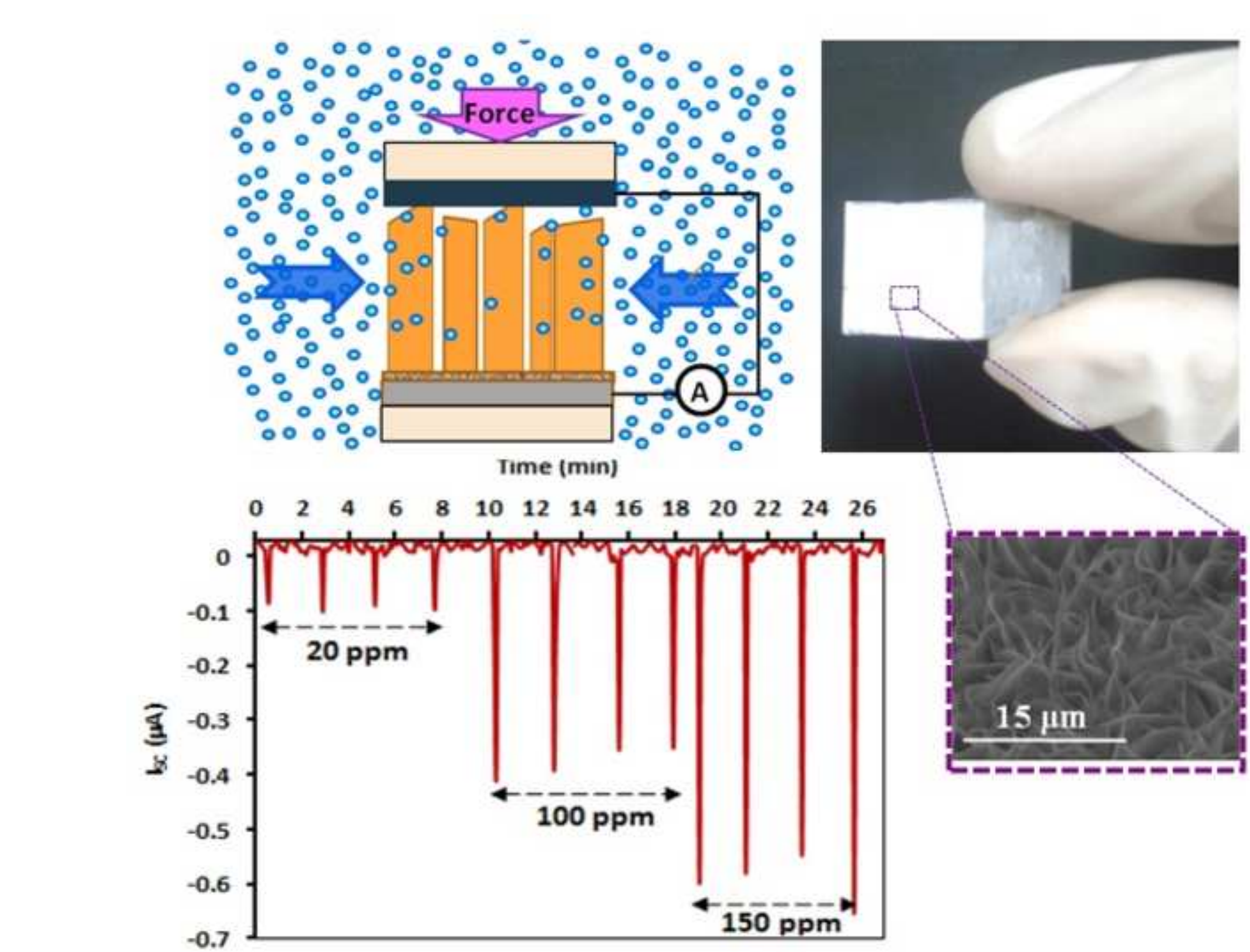

$-0.7$ 